\title{
A NEW TRAFFIC AGGREGATION SCHEME IN ALL-OPTICAL WAVELENGTH ROUTED NETWORKS
}

\author{
Nizar Bouabdallah ${ }^{1,2}$, Emannuel Dotaro $^{1}$, and Guy Pujolle ${ }^{2}$ \\ 'Alcatel Research \& Innovation, Route de Nozay, F-91460 Marcoussis, France \\ ${ }^{2}$ LIP6, University of Paris 6, 8 rue du Capitaine Scott, F-75015 Paris, France \\ e-mail: nizar.bouabdallah@lip6.fr
}

\begin{abstract}
In wavelength-division multiplexing (WDM) optical networks, the bandwidth request of a traffic stream can be much lower than the capacity of a lightpath. Efficiently grooming low-speed connections onto high-capacity lightpaths will improve the network throughput and reduce the network cost. In this paper, we propose and evaluate a new concept of traffic aggregation in mesh networks that aims to eliminate both the bandwidth underutilization and scalability problems existing in all-optical wavelength routed networks. Our objective is to improve the network throughput while preserving the benefits of all-optical wavelength routed networks.
\end{abstract}

\section{INTRODUCTION}

In the current nomenclature of optical networking, a network is referred to as transparent when its constituent nodes are all-optical cross connects (OXCs) where no conversion into the electrical domain is performed. In other words, within transparent networks, lightpaths are routed from source to destination in the optical domain, optically bypassing the intermediate nodes. Whereas, in opaque networks, lightwave channels are detected at each node, then electronically processed, switched and reassigned to a new outgoing wavelength when needed.

Realizing connections in an all-optical (transparent) wavelength routed network involves the establishment of point-to-point (PtoP) lightpaths between every edge node pair. These lightpaths may span multiple fiber links. Hence, some sort of 
virtual adjacency is created between the ingress and egress nodes via the established lightpath, even if these two nodes are geographically far apart.

In order to successfully instantiate connections, network resources (e.g., wavelengths, transceivers) have to be attributed. This issue relative to the assignment of network resources is well known as the routing and wavelength assignment (RWA) problem. A number of RWA studies have been conducted in the optical networking domain [1]-[3]. But, most previous studies assumed that a connection requests the entire bandwidth capacity of a lightpath channel. In this study, we consider the case where a connection can request either the whole or some fraction of the lightpath capacity. This makes the problem more practical and general.

The all-optical wavelength routing approach presents two obvious advantages. The first advantage stems from the fact that the optical bypass eliminates the need for Optical-Electrical-Optical (OEO) conversion at intermediate nodes. As a result, the node cost decreases significantly, since in this case the number of required expensive high-speed electronics, laser transmitters and receivers is reduced. The second advantage is due to all-optical routing which is transparent with regard to the bit rate and the format of the optical signal.

Nevertheless, wavelength routing presents two drawbacks. First, routing at a wavelength granularity puts a serious strain on the number of wavelengths required in a large network. For instance, if PtoP lightpaths needs to be established between every edge node pair in a network presenting $\mathrm{N}$ edge nodes, then $\mathrm{O}\left(\mathrm{N}^{2}\right)$ lightpaths are required. The second drawback behind wavelength routing is the rigid routing granularity entailed by such an approach. This granularity is large which could lead to bandwidth waste especially when only a portion of wavelength capacity is used. For operators, an efficient use of network resources is always a concern. In wavelength routed networks, this efficiency is possible only when there is enough traffic between pair nodes to fill the entire capacity of wavelengths.

To alleviate the aforementioned problems, we propose a new solution based on the distribution of the aggregation process. This solution combines the advantage of the optical bypass in transparent wavelength routed networks and statistical multiplexing gain in sub-wavelength routed networks. The lightpath, remaining entirely in the optical domain, is shared between the source node and all intermediate nodes up to the destination. So, we deal with multipoint-to-point (MptoP) lightpaths. Here, one lightpath represents routes from multiple ingress nodes to a single egress node.

A detailed description of this new approach will be outlined in the next section. In section 3, we investigate the node architecture needed to support trafficaggregation feature within the WDM optical network. In section 4, the comparison between distributed aggregation and the classical strategies is performed based on a simple provisioning algorithm. Finally, some conclusions are drawn in section 5 . 


\section{DISTRIBUTED AGGREGATION IN SHEME}

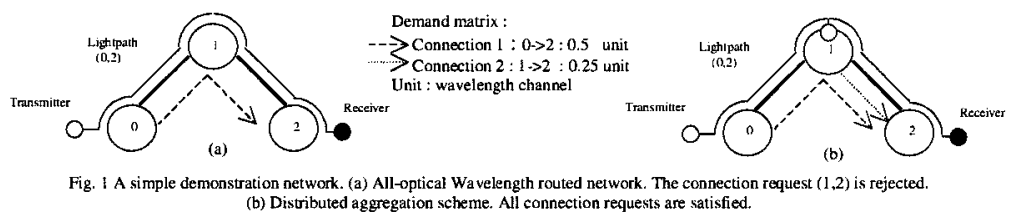

The key idea behind our proposed scheme of distributed aggregation is to allow lightpath channels sharing among several access nodes. Instead of limiting the access to lightpath capacity at the ingress point, each node along the path can fill on the fly the optical resource (wavelength) according to its availability. In this case, a lightpath channel can be shared by multiple connections traveling towards a common destination. This approach deals with MptoP connections.

Wavelength routing is performed in a similar way as in all-optical networks, i.e. signals remain in the optical domain from end to end and are optically switched by intermediate OXCs. Since the lightpath remains transparent at intermediate nodes, a MAC (Medium Access Control) protocol is required to avoid collision between transient optical packets and local ones injected into the lightpath. We have already proposed a simple MAC protocol based on void/null detection in [4]. This mechanism guarantees collision free packet insertion on the transient wavelength at the add port of an intermediate node.

To illustrate the distributed aggregation mechanism, we will present a simple three-node network example as depicted in figure. 1. Each fiber is supposed to have one wavelength channel and each node is considered as being equipped by a fixed transmitter and a fixed receiver. Two connection requests are to be served: $(0,2)$ with a bandwidth requirement equal to half of the wavelength capacity; and $(1,2)$ with a bandwidth requirement equal to quarter of the wavelength capacity. If the classical all-optical network case (i.e. networks that do not perform distributed aggregation) is taken into account (figure 1.a), only one connection $(0,2)$ would be served because of the resource limitations (the wavelength between $(1,2)$ and the receiver at node 2 are busy). The connection requested between node pair $(1,2)$ will be rejected in spite of the fact that the wavelength between these two nodes is not fully used. Hence, a supplementary wavelength between pair nodes $(1,2)$ and a new receiver at node 2 would be required in order to satisfy all the connection requests.

However, using the distributed aggregation scheme (figure1.b), the traffic demand could be satisfied by establishing one lightpath from node 0 to node 2 . In this case, both connections will share the same lightpath. Indeed, the second connection $(1,2)$ would be carried by the spare capacity of the existing lightpath. 
Note that the lightpath $0 \rightarrow 1 \rightarrow 2$ is still routed in the optical domain at node 1 , preserving the benefit of optical bypass.

The merit of distributed aggregation is that multiple connections with fractional demands can be multiplexed into the shared lightpaths. As a result, the wasted bandwidth problem confronted in pure wavelength routed networks is eliminated. In addition, due to the sharing of wavelength channels, the number of admissible connections in the network will be increased. Furthermore, as connections from different nodes to the same destination are aggregated on the same lightpath, the destination node will receive less lightpaths. So, less physical components (wavelength, transceiver) would be used, resulting in the save of a great deal of equipment costs. Moreover, in order to provide connections between all edge node pairs using MptoP lightpaths, a total number of $\mathrm{O}(\mathrm{N})$ lightpaths is required since only one lightpath per individual egress node could be sufficient. Thus, we alleviate the scalability issue encountered in all-optical wavelength routed networks. However, still additional control mechanisms would be required to arbitrate the access to the shared lightpaths.

\section{NODE ARCHITECTURE}

We consider a network of $N$ nodes connected by unidirectional optical links constituting an arbitrary physical topology. To carry connection requests in such a WDM network, lightpath connections have to be established between pairs of nodes. In all-optical networks a connection request is carried by only one lightpath before it reaches the destination in order to avoid extra signal processing at intermediate nodes along the path. Two important functionalities must be supported by the WDM network nodes: one is wavelength routing and the other is multiplexing and demultiplexing. figure. 2 depicts a sample node architecture that can be employed in a WDM optical network.

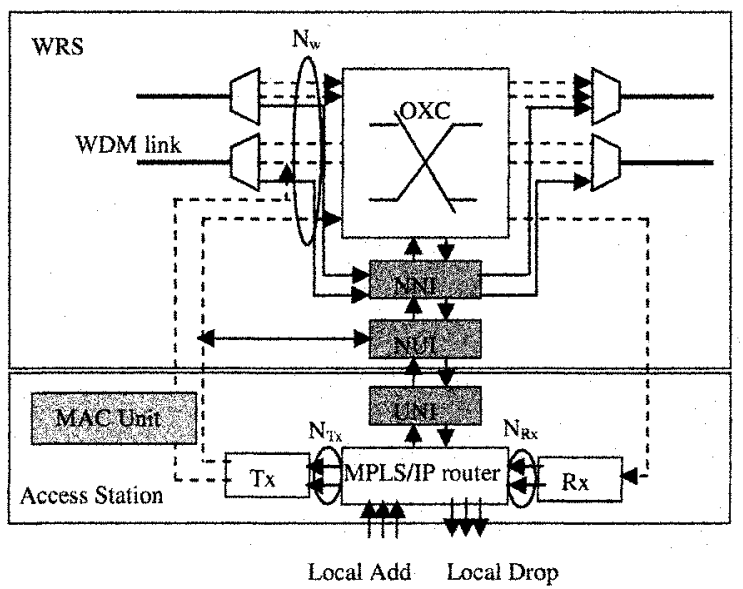

Figure. 2. Node architecture. 
The node architecture comprises two components: the wavelength-routing switch (WRS) and the access station. While the WRS performs wavelength routing and wavelength multiplexing/demultiplexing, the access station performs local traffic adding/dropping and low-speed traffic-aggregation functionalities. The WRS is composed of an OXC, which provides the functionality of wavelengthswitching.

Each OXC is connected to an edge device, i.e. access station, which can be the source or the destination of a traffic flow. In figure. 2, each access station is equipped with a certain number of transmitters and receivers. Traffic originated at the access station is transmitted as an optical signal on one wavelength channel by virtue of a transmitter. Note that the access station can be either the origin of a lightpath or an intermediate node using an already established lightpath. In the latter case, the injected traffic by an intermediate node should have the same destination as that of the traversing lightpath. Moreover, as described in [4], a MAC unit is required to avoid collision between transient packets and local ones.

Aggregating low-speed connections to high-capacity lightpaths is done by the MPLS/IP router according to the MAC unit decision. The advantages of this model are that: 1) it provides flexible bandwidth granularity for the traffic requests and 2) this MPLS/IP-over-WDM model has much less overhead than the SONET-overWDM model, widely deployed in optical networks. Usually, the potential disadvantage of such a model is that the processing speed of the MPLS/IP router may not be fast enough compared to the vast amount of bandwidth provided by the optical fiber link. However, our scheme alleviates this issue since each MPLS/IP router processes only its local traffic. In other words, the transit traffic travelling through a WDM node remains at the optical layer, and it is not processed by the intermediate access nodes.

\section{NETWORK DIMENSIONNING}

\subsection{Procedure and Algorithm for Network Dimensioning}

It is well known that the RWA optimization problem is NP-complete [5]. In [6], we used a small network topology as an illustration where it was possible to obtain results using the Integer Linear Programming (ILP) methodology. Nonetheless, in order to achieve the same study for large networks, we will use heuristic approach. In this paper, the comparison between distributed aggregation and the classical strategies is tackled from a different perspective. Here, we aim at dimensioning the optical US backbone (figure. 3), under both strategies, so as to serve all traffic requests between any pair of optical nodes. The network topology 
consists of 29 nodes and 43 links. The network planning has been achieved following the logical process shown below. The inputs of the analysis are:

1) The physical network topology.

2) The traffic matrix.

3) The adopted routing scheme, which is the shortest path algorithm in our case.

4) The adopted wavelength assignment approach, which is the first fit (FF) strategy in our work.

The network dimensioning is achieved by evaluating the OXC and IP/MPLS router dimensions by means of the heuristic algorithm used to map the different lightpaths. When dealing with the distributed aggregation strategy we will use a novel heuristic algorithm, called Maximizing Traffic Aggregation (MTA), in order to plan the MptoP lightpaths.

Let $\lambda_{s d}$ denote the aggregate traffic between node pair $s$ and $d$, which has not been yet carried. As explained before, $\lambda_{s d}$ can be a fraction of the lightpath capacity. In our study, we suppose that $\lambda_{s d} \in[0,1]$, so at most one lightpath between every node pair $(s, d)$ is required to carry all the traffic requests. Let $H(s, d)$ denote the hop distance on physical topology between node pair $(s, d)$.

The MTA algorithm attempts to establish lightpaths between sourcedestination pairs with the highest $H(s, d)$ values. The connection request between $s$ and $d$ will be carried over the new established lightpath. Afterwards, the algorithm will try to carry, as long it is possible, connections originating from intermediate nodes and travelling to the same destination $d$, based on the currently available spare capacity of the lightpath $(s, d)$. This heuristic tries to establish lightpaths between the farthest node pair in an attempt to allow the virtual topology collecting the maximum eventual traffic at the intermediate nodes. The pseudocode for this heuristic is presented hereafter:

Step 1: Construct virtual topology:

1.1: Sort all the node pairs $(s, d)$ (where $\lambda_{s d} \neq 0$ ) according to the hop distance $H(s, d)$ and insert them into a list $L$ in descending order.

1.2: Setup the lightpath between the first node pair $\left(s^{\prime}, d^{\prime}\right)$ using the first-fit wavelength assignment and the shortest-path routing, let $\lambda_{\text {s }^{\prime}{ }^{\prime}}=0$.

1.3: Sort all the node pairs $\left(i, d^{\prime}\right)$ (where $\lambda_{i d^{\prime}} \neq 0$ and $i$ is an intermediate node traversed by the lightpath $\left(s^{\prime}, d^{\prime}\right)$ ) according to the hop distance $H\left(i, d^{\prime}\right)$ and insert them into a list $L^{\prime}$ in descending order.

1.4: Try to setup the connection between the first node pair $\left(i^{\prime}, d^{\prime}\right)$ using the lightpath $\left(s^{\prime}, d^{\prime}\right)$, subject to the current available bandwidth on lightpath $\left(s^{\prime}, d^{\prime}\right)$. If it fails, delete $\left(i^{\prime}, d^{\prime}\right)$ from $L^{\prime}$; otherwise, let $\lambda_{i^{\prime} d^{\prime}}=0$, update the 
available bandwidth of the lightpath $\left(s^{\prime}, d^{\prime}\right)$ and go to step

1.3 until $L^{\prime}$ becomes empty.

1.5: Go to Step 1.1 until $L$ becomes empty.

Step 2: Route the low-speed connections over the virtual topology constructed in step 1.

The dimensioning is accomplished so that all the traffic is forwarded within the network. In the classical case, the routing algorithm is simply applied to the traffic matrix to find all the PtoP lightpaths required to forward the traffic. However, when the distributed aggregation is considered, the MTA heuristic is applied to map all the MptoP lightpaths. Then the number of OXC ports, transmitters and receivers is determined.

As explained before, the optical node consists of an electrical IP router part and an OXC part. Each port of the electrical IP router is connected to the OXC port via an internal wavelength. A lightpath is established between nodes by setting up the OXCs along the route between nodes. Some lightpaths transit to the electrical IP router after traversing the OXC part and others exit the node through the OXC without electrical processing (figure. 2). Each lightpath needs a dedicated OXC port when traversing an intermediate node along the path. In addition, a transmitter is required at the ingress node and a receiver is needed at the egress node of the lightpath. Moreover, in the distributed aggregation case, each intermediate node along the path using the traversing lightpath to transmit its traffic needs also a transmitter. Recall that, the access station of a node can be either the origin of a lightpath or an intermediate node using an already established lightpath. Let $N_{t x}$ and $N_{R x}$ denote the number of transmitter and receiver ports per node. Let $N_{w}$ denote the number of $\mathrm{OXC}$ ports per node as shown in figure. 2 .

\subsection{Dimensioning Results and Comparison}

Table 1 reports the dimensioning results of the network in the classical and the distributed aggregation cases. It is clear that each node in the network needs 28 transmitters in order to forward its traffic destined to all the other nodes in both cases. Furthermore, each node requires 28 receivers to receive all the PtoP lightpaths emanating from all the other nodes in the classical case. However, as multiple connections travelling from different nodes to the same destination could be aggregated on the same lightpath, when the distributed aggregation scheme is considered, the destination node will receive less lightpaths. Consequently the number of MptoP lightpaths and required receivers to handle all the traffic requests is reduced as depicted in table 1 . We record a save of above $55 \%$ of the receivers. 
Moreover, as the number of lightpaths is reduced whith the distributed aggregation strategy, the number of OXC ports is also reduced. The gain recorded is beyond $49 \%$. This latter gain is less important than the one obtained when dealing with receivers since the number of OXC ports depends not only on the number of established lightpaths but also on the number of hops per lightpath. Indeed, the mean hops number per lightpath is 3,58 in the classical case, whereas it is 4,2 in the other case. These results show how the distributed aggregation scheme relieves the scalability issue encountered with the classical all-optical networks.

Finally, it is meaningful to compare the lightpath load in both strategies. In the classical case, the average load of a lightpath is $27,28 \%$. This result emphasizes the resources under-utilisation problem already mentioned. Distributed aggregation scheme alleviates this issue, while the average load of a lightpath is $61,20 \%$ in this case. Figure. 4 depicts the dimensioning results corresponding to each node of the network. They represent a detailed description of the results reported in table 1.

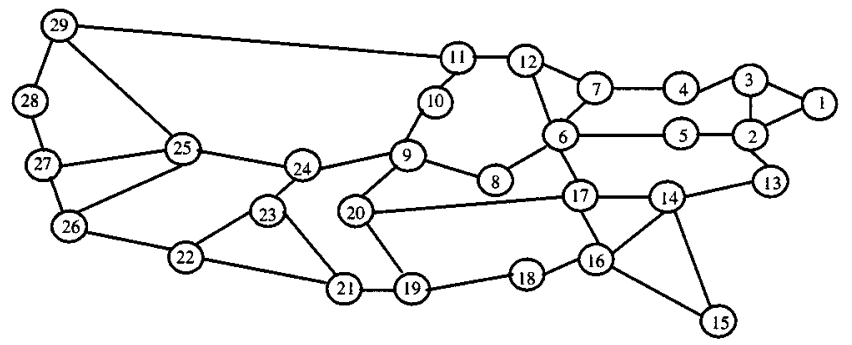

Figure. 3. The US optical backbone.

Table 1. Dimensioning results.

\begin{tabular}{|c|c|c|c|c|c|c|}
\hline & \# Tx & \# Rx & \#lightpaths & \#OXC ports & Load/ightpath & Hops/lightpath \\
\hline $\begin{array}{c}\text { Classical } \\
\text { approach }\end{array}$ & 812 & 812 & 812 & 3722 & $27,28 \%$ & 3,58 \\
\hline $\begin{array}{c}\text { Distributed } \\
\text { aggregation }\end{array}$ & 812 & 362 & 362 & 1886 & $61,20 \%$ & 4,20 \\
\hline
\end{tabular}

\section{CONCLUSIONS}

We have proposed a distributed aggregation approach in all-optical wavelength routed networks. This approach combines the merits of both the optical bypass of transparent wavelength routing and the multiplexing gain of sub-wavelength routing. In this approach, we tend to aggregate traffic traveling from different nodes to a common destination in the same lightpath channels. As a result, the number of managed lightpaths is significantly reduced and the utilization percentage of the optical channels is improved as well. The dimensioning results of 
the US optical backbone revealed that the proposed approach reduces significantly the network cost. In this specific case, around 50\% of the receiver and OXC ports are saved when the distributed aggregation is considered.

\section{REFERENCES}

[1] I. Chlamtac, A. Faragó, and T. Zhang, "Lightpath (wavelength) routing in large WDM networks", IEEE J. Select. Areas Commun., vol. 14, pp. 909-913, June 1996.

[2] D. Banerjee and B. Mukherjee, "Wavelength-routed optical networks: Linear formulation, resource budgeting tradeoffs, and a reconfiguration study", IEEE/ACM Trans. Networking, vol. 8, pp. 598-607, Oct. 2000.

[3] D. Banerjee and B. Mukherjee , "A practical approach for routing and wavelength assignment in large wavelength-routed optical networks", IEEE J. Select. Areas Commun., vol. 14, pp. 903-908, June 1996.

[4] N. Bouabdallah et al., "Matching fairness and performance by preventive traffic control in optical multiple access networks", Proc. of OptiComm '2003, Dallas, October 2003.

[5] B. Mukherjee, Optical Communication Networks. New York: Mc-Graw-Hill, 1997.

[6] N. Bouabdallah et al., "Distributed aggregation in all-optical wavelength routed networks”, ICC '2004, Paris, France, June 2004.

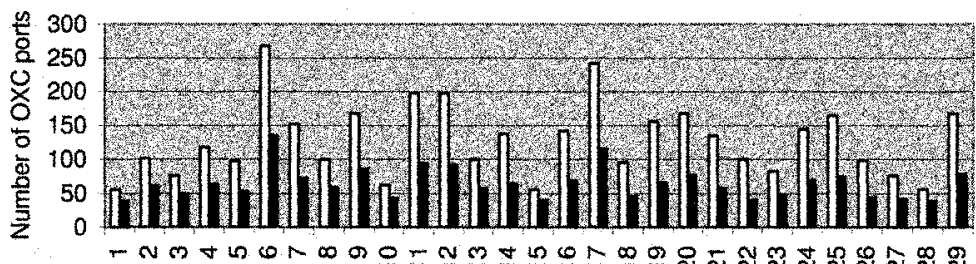

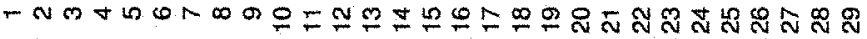

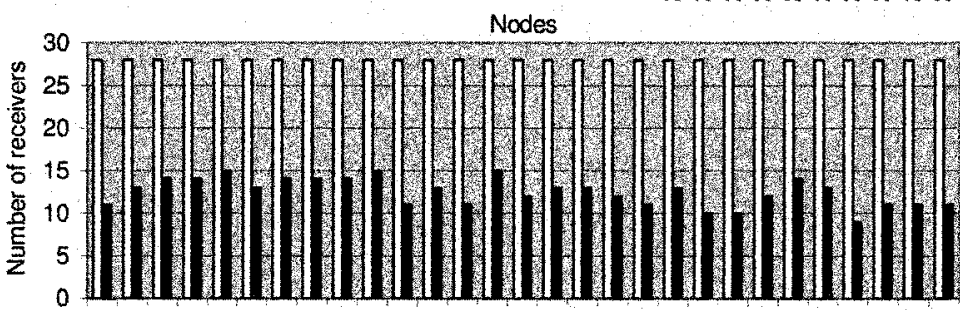

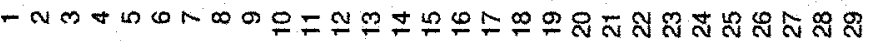

Nodes

$\square$ classical approach Distributed aggregation

Figure.4. The OXC port and receiver needs per node for the distributed aggregation and classical cases. 